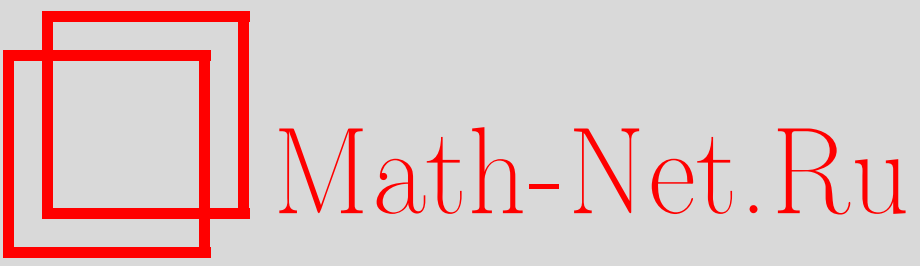

А. Н. Артемов, Термодинамика системы вихрей в тонком слоистом сверхпроводнике, ТМФ, 2000, том 125, номер 2, 326-338

DOI: https://doi.org/10.4213/tmf672

Использование Общероссийского математического портала Math-Net.Ru подразумевает, что вы прочитали и согласны с пользовательским соглашением

http://www.mathnet.ru/rus/agreement

Параметры загрузки:

IP : 34.239 .49 .27

26 апреля 2023 г., 06:26:44 
ТЕОРЕТИЧЕСКАЯ

И МАТЕМАТИЧЕСКАЯ

ФИЗИКА

Том 125, № 2

ноябрь, 2000

(C) 2000 г.

\section{ТЕРМОДИНАМИКА СИСТЕМЫ ВИХРЕЙ В ТОНКОМ СЛОИСТОМ СВЕРХПРОВОДНИКЕ}

Развит метод оценки классической статсуммы системы $N$ частиц, сводящий ее к статсумме скалярной полевой модели типа синус-Гордон. В низшем порядке теории возмущений для этой модели, что соответствует кольцевому приближению для системы частиц, найдена свободная энергия системы магнитных вихрей в тонком слоистом сверхпроводнике и исследованы ее равновесные свойства.

\section{1. ВВЕДЕНИЕ}

Слоистые структуры занимают значительное место в современной физике сверхпроводимости. К ним относятся высокотемпературные сверхпроводники, а также искусственно вырашенные слоистые системы на основе как обычных, так и высокотемпературных сверхпроводников.

Магнитное поле может проникать в сверхпроводник второго рода в виде магнитных вихрей, несуших квант магнитного потока $\phi_{0}$. Движение вихрей под действием тока приводит к джоулевым потерям и определяет резистивное поведение сверхпроводника. В настоящее время достаточно обоснованной и продуктивной является идея о возможности сушествования двумерных $(2 D)$ магнитных вихрей в слоистых сверхпроводниках. Она была высказана в работе [1]. Эти вихри имеют нормальную сердцевину только в одном слое сверхпроводника. Энергия их взаимодействия логарифмически зависит от расстояния между ними. Энергия взаимодействия вихрей, находяшихся в разных слоях, значительно меньше энергии внутрислоевого взаимодействия [2] и практически не оказывает влияния на свойства системы. В такой системе может наблюдаться фазовый переход Березинского-Костерлица-Таулесса (БКТ) [3, 4], происходящий в результате появления неустойчивости вихревых диполей относительно их диссоциации в газ свободных вихрей.

Обычно в этих структурах расстояние между сверхпроводящими слоями того же порядка, что и длина когерентности $\xi$. Это значит, что между слоями сушествует джозефсоновская (туннельная) связь. Энергия вихревого диполя, возникшего в результате

* Донецкий физико-технический институт НАН Украины, Донецк, Украина. E-mail: artemov@kinetic.ac.donetsk.ua 
тепловых флуктуаций, при этом линейно растет с ростом расстояния меж ду вихрями [5]. Она обусловлена образованием джозефсоновских вихрей, замыкающих поток вихревой пары. Наличие джозефсоновского натяжения между вихрями в диполе значительно усложняет теоретический анализ термодинамических свойств системы. Подробнее со свойствами слоистых сверхпроводников можно ознакомиться по обзорам $[6,7]$.

Реальные образцы часто выполнены в виде довольно тонких пленок и могут состоять из небольшого числа $\mathcal{N}$ слоев. Под тонким здесь подразумевается сверхпроводник, изменением тока и магнитного поля по толщине которого можно пренебречь. Магнитные вихри в однослойной пленке, удовлетворяющей этому условию, впервые рассмотрены в работе [8]. Свойства системы вихрей в тонких слоистых сверхпроводниках отличаются от свойств системы вихрей как в массивных слоистых сверхпроводниках, так и в тонких пирловских монослоях.

Отметим здесь только две особенности тонких слоистых сверхпроводников, существенных для нашего рассмотрения. Во-первых, джозефсоновскоенатяжение между вихрями в паре в таком сверхпроводнике пренебрежимо мало. Это можно понять на основании следующих соображений. Джозефсоновский вихрь образуется магнитным потоком, захваченным в промежутках между сверхпроводящими слоями. В тонком сверхпроводнике этот поток составляет очень малую часть потока джозефсоновского вихря в массивном сверхпроводнике, поскольку суммарная толщина сверхпроводящих слоев в этом случае чрезвычайно мала и они практически не экранируют поле. Эти соображения позволяют нам рассчитывать на то, что сушествуют такие слоистые сверхпроводники, свойства системы вихрей в которых можно анализировать, пренебрегая джозефсоновским натяжением. В этой работе рассматриваются только такие слоистые структуры.

Вторая особенность состоит в том, что энергия взаимодействия $2 D$-вихрей, локализованных в разных слоях, сравнима с энергией взаимодействия вихрей, расположенных в одном слое. Эти две энергии не только отличаются знаком, но имеют разные зависимости от расстояния, что создает дополнительные трудности при расчете свободной энергии системы.

Как внутрислоевое, так и межслоевое взаимодействия $2 D$-вихрей в тонких сверхпроводниках являются дальнодействуюшими. Для оценки свободной энергии такой системы в классической физике обычно разлагают ее в формальный ряд по энергии взаимодействия и суммируют главную последовательность диаграмм в кольцевом приближении [9]. В данном случае, однако, не удается собрать этот ряд таким образом, чтобы он включал оба вида взаимодействия.

Чтобы решить эту задачу, в данной статье применен метод, основанньй на введении полевой коллективной переменной, аналогичной скалярному потенциалу в системе кулоновских частиц. Таким способом статсумма системы классических частиц (вихрей) преобразуется в статсумму скалярной полевой модели типа синус-Гордон. Вычисление статсуммы полевой модели в низшем порядке теории возмушений в этом подходе соответствует кольцевому приближению для системы частиц.

Предложенным методом рассчитана свободная энергия системы вихрей тонкого слоистого сверхпроводника, состояшей из двух взаимодействуюших подсистем - $2 D$-под- 
системы и пирловской. Рассмотрена зависимость термодинамических свойств системы, в частности температуры перехода БКТ, от числа сверхпроводяших слоев, а также обсуждается влияние обеих подсистем магнитных вихрей на свойства сверхпроводника.

\section{2. МАГНИТНЫЕ ВИХРИ В ТОНКОМ СЛОИСТОМ СВЕРХПРОВОДНИКЕ}

В качестве модели рассмотрим систему $\mathcal{N}$ сверхпроводящих монослоев толщины $d$, расположенных параллельно друг другу с шагом $s$. Нас будет интересовать только пирловский [8] предел $d \ll \lambda$ для каждого слоя. Эта модель является последовательной в том случае, если не только каждый слой, но и вся система слоев пренебрежимо слабо экранирует магнитное поле. Условие, которое налагает это требование на параметры модели, получено ниже в этом разделе.

Направив ось $z$ перпендикулярно слоям, функционал свободной энергии такой системы в лондоновском пределе запишем в виде

$$
F=\frac{\phi_{0}^{2}}{16 \pi^{3} \Lambda} \sum_{n=1}^{\mathcal{N}} \int d \mathbf{r}\left(\nabla \theta_{n}(\mathbf{x})-\frac{2 \pi}{\phi_{0}} \mathbf{A}(\mathbf{r})\right)^{2} \delta(z-n s)+\int d \mathbf{r} \frac{(\operatorname{rot} \mathbf{A})^{2}}{8 \pi},
$$

где $\Lambda=2 \lambda^{2} / d, \nabla \theta_{n}(\mathbf{x})$ - градиент фазы параметра порядка в слое $n, \mathbf{A}(\mathbf{r})$ - векторный потенциал, $\mathbf{r}=(\mathbf{x}, z)$.

Приравнивая к нулю вариацию функционала (1) по А, получим уравнения равновесия системы

$$
\begin{gathered}
\operatorname{rot} \operatorname{rot} \mathbf{A}=\frac{4 \pi}{c} \sum_{n=1}^{\mathcal{N}} \mathbf{I}_{n}(\mathbf{x}), \\
\frac{4 \pi}{c} \mathbf{I}_{n}(\mathbf{x})=\frac{2}{\Lambda}\left[\frac{\phi_{0}}{2 \pi} \nabla \theta_{n}(\mathbf{x})-\mathbf{A}(\mathbf{r})\right] \delta(z-n s) .
\end{gathered}
$$

Эти уравнения нужно дополнить калибровочными условиями

$$
\operatorname{div} \mathbf{A}=0, \quad \operatorname{div} \nabla \theta=0
$$

и уравнениями для градиента фазы параметра порядка, учитываюшими особенность в точках, соответствующих положению кора вихрей,

$$
\operatorname{rot} \nabla \theta\left(\mathbf{x}-\mathbf{x}_{i}\right)= \pm 2 \pi \hat{z} \delta\left(\mathbf{x}-\mathbf{x}_{i}\right) .
$$

Здесь $\hat{z}$ - единичный вектор в направлении оси $z, \mathbf{x}_{i}$ - координата $i$-го вихря, знаки \pm в правой части формулы (4) соответствуют двум возможным направлениям магнитного потока вихря.

Используя уравнения равновесия, получим выражение для равновесной энергии системы

$$
F=\frac{\phi_{0}}{4 \pi c} \sum_{n=1}^{\mathcal{N}} \int d \mathbf{r}\left(\mathbf{I}_{n}(\mathbf{r}), \nabla \theta_{n}(\mathbf{x})\right)=\frac{\phi_{0}}{4 \pi c} \sum_{n=1}^{\mathcal{N}} \int \frac{d \mathbf{q}}{(2 \pi)^{2}}\left(\mathbf{I}_{n}(\mathbf{q}), \nabla \theta_{n}(-\mathbf{q})\right)
$$


Как видно из (5), равновесная энергия системы выражается через ток и градиент фазы, локализованные в сверхпроводяших слоях. Таким образом, чтобы вычислить энергию одного вихря или энергию взаимодействия двух вихрей, нужно найти созданные ими токи и градиенты фазы.

Распределение градиента фазы в слое дается решением уравнения (4). Уравнения для тока легко получить из (2) и (3) при помощи преобразования Фурье. Выразив векторный потенциал в $n$-м слое из (2)

$$
\mathbf{A}_{n}(\mathbf{q})=\int_{-\infty}^{\infty} \frac{d k}{2 \pi} \mathbf{A}(\mathbf{q}, k) e^{i k n s}=\frac{4 \pi}{c} \sum_{m=1}^{\mathcal{N}} \mathbf{I}_{m}(\mathbf{q}) \frac{e^{-q s|n-m|}}{2 q}
$$

и подставив его в (3), найдем

$$
\mathbf{I}_{n}(\mathbf{q})+\sum_{m=1}^{\mathcal{N}} \mathbf{I}_{m}(\mathbf{q}) \frac{e^{-q s|n-m|}}{\Lambda q}=\frac{\phi_{0} c}{4 \pi^{2} \Lambda} \nabla \theta_{n}(\mathbf{q})
$$

Полученная система уравнений может быть значительно упрощена в пирловском пределе, который означает пренебрежение экранированием магнитного поля тонкой сверхпроводяшей пленкой. В соответствии с этим при $\mathcal{N}>1$ нужно заменить единицами все экспоненты в уравнениях (6) для тока, что можно сделать при условии

$$
\mathcal{N} s \ll \Lambda .
$$

Это условие можно получить, вычисляя энергию $2 D$-вихря для $\mathcal{N}=2,3$, когда система (6) легко решается без упрошений. Оно значительно слабее условия, налагаемого на пирловскую пленку, и ему, по-видимому, удовлетворяет большинство пленок на основе высокотемпературных сверхпроводников. Это можно понять, поскольку значительную часть объема слоистого сверхпроводника могут занимать несверхпроводящие прослойки. Здесь нужно уточнить, что условие (7) позволяет нам только упростить уравнения (6), но не оценивает возможность пренебречь джозефсоновским натяжением. При $\mathcal{N}=1$ наше рассмотрение справедливо в обычном приближении тонких сверхпроводящих пленок $d \ll \lambda$.

При указанных ограничениях система уравнений (6) сведется к системе

$$
\mathbf{I}_{n}(\mathbf{q}) \Lambda q+\sum_{m=1}^{\mathcal{N}} \mathbf{I}_{m}(\mathbf{q})=\frac{\phi_{0} c}{4 \pi^{2}} q \nabla \theta_{n}(\mathbf{q})
$$

Энергия вихря в этом случае не зависит от номера слоя, в котором он расположен. Для определенности будем считать, что вихрь находится в первом слое. При этом отлична от нуля будет правая часть только первого уравнения в (8). Легко видеть, что система уравнений удовлетворяется, если $\mathbf{I}_{2}=\mathbf{I}_{3}=\cdots=\mathbf{I}_{\mathcal{N}}$. Для $\mathbf{I}_{1}(\mathbf{q})$ получаем

$$
\mathbf{I}_{1}(\mathbf{q})=\frac{\phi_{0} c}{4 \pi^{2} \Lambda} \frac{\mathcal{N}-1+\Lambda q}{\mathcal{N}+\Lambda q} \nabla \theta_{1}(\mathbf{q})
$$


Подставляя в (5) полученное выражение для тока и для градиента фазы, найдем энергию $2 D$-вихря в $\mathcal{N}$-слойном сверхпроводнике

$$
F_{\mathcal{N}}=\frac{\phi_{0}^{2}}{4 \pi \Lambda} \int \frac{d \mathbf{q}}{(2 \pi)^{2}} \frac{1}{q^{2}}\left(1-\frac{1}{\mathcal{N}+\Lambda q}\right)=\frac{\phi_{0}^{2}}{8 \pi^{2} \Lambda}\left[\left(1-\frac{1}{\mathcal{N}}\right) \ln \frac{R}{\xi}+\frac{1}{\mathcal{N}} \ln \frac{\Lambda}{\mathcal{N} \xi}\right] .
$$

Первое слагаемое в квадратных скобках логарифмически расходится на нижнем пределе $q=0$ и обрезано размером образца $R$. Оно характерно для $2 D$-вихрей. Второе слагаемое подобно энергии пирловского вихря [8].

Аналогично вычисляется энергия взаимодействия двух $2 D$-вихрей, расположенных в одном или разных слоях,

$$
\begin{aligned}
U_{m n}(l) & =\mp \frac{\phi_{0}^{2}}{2 \pi \Lambda} \int \frac{d \mathbf{q}}{(2 \pi)^{2}} \frac{1}{q}\left[\left(\delta_{m n}-\frac{1}{\mathcal{N}}\right) \frac{1}{q}+\frac{1}{\mathcal{N}} \frac{1}{q+\mathcal{N} / \Lambda}\right] \cos (\mathbf{q} \mathbf{l})= \\
& = \pm \frac{\phi_{0}^{2}}{4 \pi^{2} \Lambda}\left\{\left(\delta_{m n}-\frac{1}{\mathcal{N}}\right) \ln \frac{l}{\xi}-\frac{\pi}{2 \mathcal{N}}\left[\mathbf{H}_{0}\left(\frac{\mathcal{N} l}{\Lambda}\right)-Y_{0}\left(\frac{\mathcal{N} l}{\Lambda}\right)\right]\right\} .
\end{aligned}
$$

Индексы $m$ и $n$ соответствуют номерам слоев, в которых расположены взаимодействующие вихри. Верхний и нижний знаки перед выражениями соответствуют парам вихрей, магнитные потоки которых ориентированы соответственно в противоположном и одинаковом направлениях. Энергия (11) также имеет черты, характерные как для 2D-, так и для пирловских вихрей.

Как видно из приведенного выражения, энергия взаимодействия вихрей, расположенных в разных слоях, пропорциональна $1 / \mathcal{N}$. При $\mathcal{N}=2$ она практически равна энергии внутрислоевого взаимодействия и убывает с увеличением числа слоев.

Кроме $2 D$-вихрей в системе из $\mathcal{N}>1$ сверхпроводяших слоев могут сушествовать связанные состояния $2 D$-вихрей, находяшихся в разных (не обязательно соседних) слоях. Поскольку рассматриваемая система линейна, их собственные энергии и энергии взаимодействия равны суммам соответствующих энергий $2 D$-вихрей. Мы не будем рассматривать влияние большинства таких образований на поведение системы, поскольку они имеют значительно большую энергию и концентрация их мала при любых температурах. Исключение сделаем только для связанных состояний из $\mathcal{N} 2 D$-вихрей, расположенных друг над другом в разных слоях и пронизывающих весь сверхпроводник. В отличие от $2 D$-вихря, энергия такого связанного состояния конечна и равна энергии пирловского вихря в пленке толшиной $\mathcal{N} d$ с эффективной длиной экранирования $\Lambda / \mathcal{N}$,

$$
F_{\mathrm{P}}=\frac{\phi_{0}^{2} \mathcal{N}}{8 \pi^{2} \Lambda} \ln \frac{\Lambda}{\mathcal{N} \xi}
$$

Конечная, хотя и малая, концентрация таких вихрей присутствует в сверхпроводнике при любой отличной от нуля температуре. Поэтому эти вихри определяют поведение системы в области низких температур, где другие вихри отсутствуют.

В дальнейшем нам удобно рассматривать взаимодействие (11) двух $2 D$-вихрей как потенциальное взаимодействие частиц с эффективными зарядами $Q= \pm \phi_{0} /(2 \pi \sqrt{\Lambda})$,

$$
U_{m n}\left(\mathbf{x}_{i}-\mathbf{x}_{j}\right)=-Q_{i m} Q_{j n} u_{m n}\left(\mathbf{x}_{i}-\mathbf{x}_{j}\right) .
$$


При помощи преобразования Фурье легко установить, что потенциал $u_{m n}\left(\mathbf{x}_{i}-\mathbf{x}_{j}\right)$ удовлетворяет уравнению

$$
-\Delta u_{m n}(\mathbf{x})+\sum_{k=1}^{\mathcal{N}} \int d \mathbf{y} K_{m k}(\mathbf{x}-\mathbf{y}) u_{k n}(\mathbf{y})=2 \pi \delta_{m n} \delta(\mathbf{x})
$$

с ядром

$$
K_{m k}(\mathbf{x})=\int \frac{d \mathbf{q}}{(2 \pi)^{2}} \frac{q}{\Lambda} e^{i(\mathbf{q} \mathbf{x})}
$$

Первый член этого уравнения дает локальную кулоновскую часть взаимодействия, а второй - нелокальную, характерную для пирловских вихрей.

\section{3. ОЦЕНКА СВОБОДНОЙ ЭНЕРГИИ СИСТЕМЫ}

Целью нашего рассмотрения являются термодинамические свойства системы взаимодействующих $2 D$ - и пирловских вихрей в $\mathcal{N}$-слойном сверхпроводнике. Это система с переменным числом частиц. Равновесная концентрация частиц в ней определяется из условий минимума свободной энергии системы. Без внешнего магнитного поля числа вихрей с положительным $N_{+}$и отрицательным $N_{-}$направлениями магнитного потока равны, и мы будем использовать для них одинаковые обозначения.

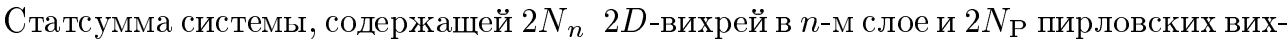
рей, может быть записана в виде

$$
Z\left[\left\{N_{n}\right\}, N_{\mathrm{P}}\right]=\prod_{n=1}^{\mathcal{N}} \frac{1}{\left(N_{n} !\right)^{2}} \frac{1}{\left(N_{\mathrm{P}} !\right)^{2}} \operatorname{Sp} \exp \left\{-\beta H-2\left(N_{n}+\mathcal{N} N_{\mathrm{P}}\right) \beta E_{0}\right\}
$$

где $E_{0}$ - энергия кора вихря, $\beta=1 / T$, операция Sp означает суммирование по всем состояниям вихрей в системе,

$$
\mathrm{Sp}=\prod_{n=1}^{\mathcal{N}}\left(e^{-\beta E_{0}} \int \frac{d \mathbf{x}_{j n}}{\pi \xi^{2}}\right)^{2 N_{n}}\left(e^{-\beta \mathcal{N} E_{0}} \int \frac{d \mathbf{y}_{j_{\mathrm{P}}}}{\pi \xi^{2}}\right)^{2 N_{\mathrm{P}}}
$$

Гамильтониан системы вихрей определяется выражением

$$
\begin{aligned}
H= & \frac{1}{2} \sum_{m n} \sum_{i_{m}, j_{n}} Q_{i_{m}} Q_{j_{n}} u_{m n}\left(\mathbf{x}_{i_{m}}-\mathbf{x}_{j_{n}}\right)+\frac{1}{2} \sum_{i_{\mathrm{P}} j_{\mathrm{P}}} Q_{i_{\mathrm{P}}} Q_{j_{\mathrm{P}}} v\left(\mathbf{y}_{i_{\mathrm{P}}}-\mathbf{y}_{j_{\mathrm{P}}}\right)+ \\
& +\sum_{m} \sum_{i_{m} j_{\mathrm{P}}} Q_{i_{m}} Q_{j_{\mathrm{P}}} w_{m}\left(\mathbf{x}_{i_{m}}-\mathbf{y}_{j_{\mathrm{P}}}\right) .
\end{aligned}
$$

Суммирование по индексам вихрей $(i, j)$ в каждой сумме производится независимо по всем вихрям. Слагаемые с равными индексами дают собственные энергии вихрей, а с различными - энергии взаимодействия $2 D$-вихрей $u_{m n}$, пирловских вихрей $v$ и энергию 
взаимодействия $2 D$ - и пирловских вихрей $w_{m}$. Поскольку пирловский вихрь представляется как связанное состояние $2 D$-вихрей, потенциалы взаимодействия с пирловскими вихрями могут быть представлены в виде суммы потенциалов $2 D$-вихрей, т.е.

$$
\begin{aligned}
v\left(\mathbf{y}_{i_{\mathrm{P}}}-\mathbf{y}_{j_{\mathrm{P}}}\right) & =\sum_{m n} u_{m n}\left(\mathbf{y}_{i_{\mathrm{P}}}-\mathbf{y}_{j_{\mathrm{P}}}\right), \\
w_{m}\left(\mathbf{x}_{i_{m}}-\mathbf{y}_{j_{\mathrm{P}}}\right) & =\sum_{n} u_{m n}\left(\mathbf{x}_{i_{m}}-\mathbf{y}_{j_{\mathrm{P}}}\right) .
\end{aligned}
$$

В рассматриваемой системе вихрей есть два вида взаимодействия между вихрями: внутри- и межслоевое. Как видно из (11), они отличаются знаком и по величине. Для оценки свободной энергии системы таких частиц мы применили прием, использованный в работе [10] (см. также [11]) для расчета термодинамических свойств кулоновской плазмы. Здесь мы развили формализм для оценки классической статсуммы и рассмотрели более общий случай межчастичных взаимодействий.

Чтобы преобразовать статсумму (15), умножим ее на единищу, представленную в виде функционального интеграла по скалярной переменной $\phi$,

$$
1=A^{-1} \int \mathrm{D} \phi \exp \left\{-\frac{\beta}{2} \sum_{m n} \phi_{m} \widehat{M}_{0 m n} \phi_{n}\right\}
$$

Действие оператора $\widehat{M}_{0}=\left(\nabla^{2}+\widehat{K}\right) /(2 \pi)$ на функции $\phi$ определяется выражением

$$
\phi_{m} \widehat{M}_{0 m n} \phi_{n}=\frac{1}{2 \pi} \int d \mathbf{y} d \mathbf{y}^{\prime}\left[\left(\nabla \phi_{m}(\mathbf{y})\right)^{2} \delta_{m n} \delta\left(\mathbf{y}-\mathbf{y}^{\prime}\right)+\phi_{m}(\mathbf{y}) K_{m n}\left(\mathbf{y}-\mathbf{y}^{\prime}\right) \phi_{n}\left(\mathbf{y}^{\prime}\right)\right] .
$$

Нормировочная постоянная $A$ равна континуальному интегралу и формально может быть выражена через определитель оператора $\widehat{M}_{0}$ как $A=\left(\operatorname{det} \widehat{M}_{0}\right)^{-1 / 2}$. Оператор $\widehat{K}$ здесь тот же, что и в уравнении (14).

Сделаем в интеграле по ф замену переменных

$$
\phi_{n}(\mathbf{y}) \Rightarrow \phi_{n}(\mathbf{y})+i \sum_{m} \sum_{i_{m}} Q_{i_{m}} u_{m n}\left(\mathbf{y}-\mathbf{x}_{i_{m}}\right)+i \sum_{m} \sum_{j_{\mathrm{P}}} Q_{j_{\mathrm{P}}} u_{m n}\left(\mathbf{y}-\mathbf{y}_{j_{\mathrm{P}}}\right) .
$$

Она подобрана так, чтобы после подстановки в (16) сократились члены с потенциалом взаимодействия в аргументе экспоненты в выражении (15) для статсуммы. Для этого нужно в слагаемых с градиентом потенциала $u_{m n}$ интегрированием по частям перебросить вторую производную на этот потенциал и использовать уравнение (14) для получения дельта-функции, снимающей интегрирование. В результате выражение для статсуммы преобразуется к виду

$$
Z\left[\left\{N_{n}\right\}, N_{\mathrm{P}}\right]=A^{-1} \int \mathrm{D} \phi \exp \left\{-\beta S_{0}[\phi]\right\} Z\left[\left\{N_{n}\right\}, N_{\mathrm{P}}, \phi\right]
$$

где

$$
Z\left[\left\{N_{n}\right\}, N_{\mathrm{P}}, \phi\right]=\operatorname{Sp} \exp \left\{-\beta S_{1}[\phi]\right\}
$$


- статсумма вихрей, взаимодействуюших со скалярным полем $\phi$, а эффективное действие системы дается выражением

$S[\phi]=S_{0}[\phi]+S_{1}[\phi]=\frac{1}{2} \sum_{m n} \phi_{m} \widehat{M}_{0 m n} \phi_{n}+i \sum_{m}\left(\sum_{i_{m}} Q_{i_{m}} \phi_{m}\left(\mathbf{x}_{i_{m}}\right)+\sum_{i_{\mathrm{P}}} Q_{i_{\mathrm{P}}} \phi_{m}\left(\mathbf{y}_{i_{\mathrm{P}}}\right)\right)$

Оценим свободную энергию системы вихрей в поле $\phi$. Для этого разложим ее в формальный ряд по взаимодействию. Известно, что логарифм статсуммы разлагается в ряд по связным диаграммам [9]. В данном случае связными будут только те члены ряда, все сомножители которых относятся к одному и тому же вихрю. Конфигурационная часть свободной энергии разлагается в ряд по четным степеням $\phi$. Нечетные члены исчезают, поскольку суммарный заряд системы равен нулю, таким образом, имеем

$$
\begin{aligned}
-\beta E_{\mathrm{c}}[\phi]= & \sum_{m} \sum_{j_{m}} \int \frac{d \mathbf{x}_{j_{m}}}{S} \sum_{b=1}^{\infty} \frac{(-1)^{b}}{(2 b) !}\left(\beta Q_{j_{m}}\right)^{2 b} \phi_{m}^{2 b}\left(\mathbf{x}_{j_{m}}\right)+ \\
& +\sum_{m n} \sum_{j_{\mathrm{P}}} \int \frac{d \mathbf{y}_{j_{\mathrm{P}}}}{S} \sum_{b=1}^{\infty} \frac{(-1)^{b}}{(2 b) !}\left(\beta Q_{j_{m}}\right)^{2 b}\left(\phi_{m}\left(\mathbf{y}_{j_{\mathrm{P}}}\right) \phi_{n}\left(\mathbf{y}_{j_{\mathrm{P}}}\right)\right)^{b} .
\end{aligned}
$$

Результат интегрирования по координате не зависит от индекса вихря. Поэтому суммы по $j_{m}$ и $j_{\mathrm{P}}$ дают просто числа $2 D$ - и пирловских вихрей, а сумма по $b$ "сворачивается" в косинус. Окончательно получаем

$$
\begin{aligned}
-\beta E_{\mathrm{c}}[\phi]= & 2 \sum_{m n} \int \frac{d \mathbf{x}}{S}\left[N_{m}\left(\cos \left(\beta Q \phi_{m}(\mathbf{x})\right)-1\right) \delta_{m n}+\right. \\
& \left.\left.+N_{\mathrm{P}}\left(\cos \left(\beta Q \sqrt{\phi_{m}(\mathbf{x}) \phi_{n}(\mathbf{x}}\right)\right)-1\right)\right] .
\end{aligned}
$$

Таким образом, подставляя в (18) статсумму вихрей в поле $\phi$ с конфигурационной энергией (19), получим статсумму скалярной полевой модели типа синус-Гордон. Для кулоновского газа такая процедура приводит к хорошо известному его соответствию скалярной модели синус-Гордон [12] (см. также [6]).

Для вычисления интеграла по $\phi$ можно использовать теорию возмушений. Используя $S_{0}$ в качестве невозмушенного действия, мы получим обычную теорию возмушений, содержашую кулоновские расходимости, связанные с медленным убыванием взаимодействия вихрей на больших расстояниях. Использованный здесь прием позволяет переформулировать теорию возмушений, присоединив к невозмушенному действию квадратичную по ф часть коррелящионной энергии (19). Новая теория возмушений, использующая перенормированное невозмущенное действие

$$
\widetilde{S}_{0}[\phi]=\frac{1}{2} \sum_{m n} \phi_{m}\left(\widehat{M}_{0 m n}+\widehat{M}_{1 m n}\right) \phi_{n}=\frac{1}{2} \sum_{m n} \phi_{m} \widehat{M}_{m n} \phi_{n}
$$


свободна от кулоновских расходимостей. Матрица $\widehat{M}_{m n}$ определяется структурой квадратичной части $E_{\mathrm{c}}$ :

$$
M_{1 m n}=2 \frac{N_{m}}{S} \beta Q^{2} \delta_{m n}+\left(2 \frac{N_{\mathrm{P}}}{S} \beta Q^{2}\right)_{m n}
$$

Мы ограничим наше рассмотрение наинизшим приближением для свободной энергии, учитывая только квадратичные по ф слагаемые в эффективном действии, которые составляют $\widetilde{S}_{0}[\phi]$. В этом приближении интеграл по $\phi$ легко вычисляется в представлении Фурье, в котором оператор $\widehat{M}$ диагонален. В результате выражение для конфигурационной энергии представляется в виде

$$
\beta E_{\mathrm{c}}=\frac{1}{2} \sum_{\mathbf{q}} \ln \operatorname{det}\left(\widehat{M}(\mathbf{q}) \widehat{M}_{0}^{-1}(\mathbf{q})\right)
$$

Матрица $\widehat{M}_{0}^{-1}$ под знаком определителя появилась из нормировочной постоянной $A$.

Выражение (22) обобщает кольцевое приближение на случай вихрей в слоистых сверхпроводниках, при этом учитьвается не только внутрислоевое, но и межслоевое взаимодействие. Для кулоновской плазмы оно приводит к известным результатам [9].

\section{4. ТЕРМОДИНАМИКА СИСТЕМЫ ВИХРЕЙ}

Количество термодинамических переменных системы вихрей зависит от числа сверхпроводящих слоев, поскольку концентрации $2 D$-вихрей в них независимы. Достаточно очевидно, однако, что равновесная конщентрация вихрей одинакова во всех слоях. Поэтому, интересуясь только равновесньми свойствами системы, мы можем сократить число термодинамических переменных задачи до двух: концентрации $2 D$-вихрей (антивихрей) в одном слое $N$ и концентрации пирловских вихрей $N_{\mathrm{P}}$. В этом случае все результаты могут быть получены в общем виде.

В соответствии с (17) и (21) вычисление определителя матрицы $\widehat{M} \widehat{M}_{0}^{-1}$ в представлении Фурье приводит к выражению

$$
\ln \operatorname{det}\left(\widehat{M} \widehat{M}_{0}^{-1}\right)=(\mathcal{N}-1) \ln \left(1+\frac{1}{\delta^{2} q^{2}}\right)+\ln \left(1+\frac{1}{\delta_{1}^{2} q\left(q+\frac{\mathcal{N}}{\Lambda}\right)}\right),
$$

где $\delta^{-2}=4 \pi Q^{2} \beta N / S$ и $\delta_{1}^{-2}=4 \pi Q^{2} \beta\left(N / S+\mathcal{N} N_{\mathrm{P}} / S\right)$ - две длины экранирования, что связано с наличием в системе двух видов взаимодействия между частицами: дальнодействуюшего кулоновского и пирловского, ограниченного эффективной длиной $\Lambda / \mathcal{N}$.

Выполняя интегрирование по q в выражении для конфигурационной энергии, мы обрезаем интеграл сверху значением $q=1 / \xi$, что является стандартным способом устранения ультрафиолетовой расходимости в лондоновской электродинамике. Вводя безразмерные обозначения $f=\beta F \pi \xi^{2} / S$ для свободной энергии системы и $n=N \pi \xi^{2} / S$ 
для концентрации вихрей, получим

$$
\begin{aligned}
f= & 2 n(\ln n-1)+\frac{2 n_{\mathrm{P}}}{\mathcal{N}}\left(\ln n_{\mathrm{P}}-1\right)+2 p\left(n+n_{\mathrm{P}}\right) e_{0}+ \\
& +2 p n\left(1-\frac{1}{\mathcal{N}}\right)(1-\ln 16 p n)+\frac{2 p\left(n+\mathcal{N} n_{\mathrm{P}}\right)}{\mathcal{N}}\left(1-\ln 16 p\left(n+\mathcal{N} n_{\mathrm{P}}\right)\right)+ \\
& +\frac{\mathcal{N}}{8 \Lambda^{2}} G\left(64 p\left(n+\mathcal{N} n_{\mathrm{P}}\right) \frac{\Lambda^{2}}{\mathcal{N}^{2}}\right) .
\end{aligned}
$$

Здесь введено обозначение $p=\phi_{0}^{2} /\left(16 \pi^{2} \Lambda T\right), \Lambda$ измеряется в единицах $\xi$, а функция $G$ определена выражением

$$
G(x)=\frac{1}{2} \ln \frac{x}{4}+\sqrt{|1-x|} \begin{cases}\operatorname{arctg} \frac{1}{\sqrt{x-1}}-\frac{\pi}{2}, & x \geqslant 1, \\ \frac{1}{2} \ln \frac{1+\sqrt{1-x}}{1-\sqrt{1-x}}, & x \leqslant 1 .\end{cases}
$$

Равновесные концентрации вихрей в системе определяются из условий минимума свободной энергии. Они подчиняются системе уравнений

$$
\begin{gathered}
\left(1-p\left(1-\frac{1}{\mathcal{N}}\right)\right) \ln n-\frac{p}{\mathcal{N}} \ln \left(n+\mathcal{N} n_{\mathrm{P}}\right)+ \\
+\frac{4 p}{\mathcal{N}} G^{\prime}\left(64 p\left(n+\mathcal{N} n_{\mathrm{P}}\right) \frac{\Lambda^{2}}{\mathcal{N}^{2}}\right)=p\left(\ln 16 p-e_{0}\right), \\
\frac{1}{\mathcal{N}} \ln n_{\mathrm{P}}-p \ln \left(n+\mathcal{N} n_{\mathrm{P}}\right)+4 p G^{\prime}\left(64 p\left(n+\mathcal{N} n_{\mathrm{P}}\right) \frac{\Lambda^{2}}{\mathcal{N}^{2}}\right)=p\left(\ln 16 p-e_{0}\right) .
\end{gathered}
$$

Сначала исследуем две важные асимптотики решения этой системы. В случае предельно низких концентраций вихрей $n \ll n_{\mathrm{P}} \ll(\mathcal{N} / \Lambda)^{2}$ система уравнений $(26)$ значительно упрошается,

$$
\begin{aligned}
\left(1-p\left(1-\frac{1}{\mathcal{N}}\right)\right) \ln n & =p\left(\left(1-\frac{1}{\mathcal{N}}\right) \ln 16 p-\frac{1}{\mathcal{N}} \ln \left(\frac{\Lambda}{\mathcal{N}}\right)^{2}-e_{0}\right), \\
\ln n_{\mathrm{P}} & =\mathcal{N} p\left(\ln \left(\frac{\mathcal{N}}{\Lambda}\right)^{2}-e_{0}\right) .
\end{aligned}
$$

Подсистемы $2 D$ - и пирловских вихрей в этом пределе независимы друг от друга. Решение первого уравнения дает концентрацию $2 D$-вихрей

$$
n=\left[\frac{(16 p)^{1-\frac{1}{\mathcal{N}}}}{e^{e_{0}}}\left(\frac{\mathcal{N}}{\Lambda}\right)^{\frac{2}{\mathcal{N}}}\right]^{\frac{p}{1-p\left(1-\frac{1}{\mathcal{N}}\right)}} .
$$

Оно отшепляется от решения $n=0$ при $p(1-1 / \mathcal{N})=1$, что соответствует температуре

$$
T_{\mathrm{KT}}=\frac{\phi_{0}^{2}}{16 \pi^{2} \Lambda\left(T_{\mathrm{KT}}\right)}\left(1-\frac{1}{\mathcal{N}}\right)
$$


При этой температуре в подсистеме $2 D$-вихрей происходит фазовый переход БКТ $[3,4]$. Выше $T_{\mathrm{KT}}$ в сверхпроводнике появляется конечная концентрация $2 D$-вихрей и становится конечной длина экранирования $\delta$, которая является корреляционной длиной для кулоновской части взаимодействия $2 D$-вихрей. Полученные решения справедливы при условии $\infty>\delta \gg \Lambda / \mathcal{N}$, которое показывает, что экранируется только кулоновская часть энергии взаимодействия $2 D$-вихрей. Логарифмическая расходимость в энергии $2 D$-вихря при этом обрезается длиной экранирования, энергия $2 D$-вихря становится конечной и начинает зависеть от концентрации $2 D$-вихрей. В результате равновесная концентрация их лавинообразно нарастает.

Явную зависимость температуры $T_{\mathrm{KT}}$ от числа слоев можно получить, если известна зависимость $\Lambda(T)$. Полагая, что вблизи $T_{\mathrm{KT}}$ эта зависимость имеет вид $\Lambda(T) \sim$ $\Lambda(0) /\left(1-T / T_{\mathrm{c}}\right)$, получим

$$
T_{\mathrm{KT}}(\mathcal{N})=\frac{T_{\infty}}{1+\frac{1}{\mathcal{N}-1}\left(1-\frac{T_{\infty}}{T_{\mathrm{c} 0}}\right)}
$$

где $T_{\mathrm{c} 0}$ - температура перехода в сверхпроводящее состояние, $T_{\infty}=T_{\mathrm{KT}}(\infty)$. Таким образом, температура $T_{\mathrm{KT}}(\mathcal{N})$ является монотонной функцией числа слоев. Она равна нулю при $\mathcal{N}=1$ и асимптотически приближается к $T_{\infty}$ при увеличении числа слоев.

Второе уравнение системы (27) приводит к обычному больцмановскому выражению для концентрации пирловских вихрей с энергией (12)

$$
n_{\mathrm{P}}=\exp \left\{-\beta\left(F_{\mathrm{P}}+\mathcal{N} E_{0}\right)\right\}=\left(\left(\frac{\mathcal{N}}{\Lambda}\right)^{2} e^{-e_{0}}\right)^{\mathcal{N} p}
$$

В противоположном пределе $n \gg n_{\mathrm{P}} \gg(\mathcal{N} / \Lambda)^{2}$ система уравнений равновесия сводится к системе

$$
\begin{aligned}
(1-p) \ln n & =p\left(\ln 16 p-e_{0}\right), \\
\frac{1}{\mathcal{N}} \ln n_{\mathrm{P}}-p \ln n & =p\left(\ln 16 p-e_{0}\right) .
\end{aligned}
$$

Эта асимптотика выявляет еще одну неустойчивость в системе вихрей, которая возникает при $p=1\left(T=T_{\infty}\right)$. Концентрации как $2 D$-, так и пирловских вихрей резко возрастают с температурой при переходе через эту точку,

$$
n=\left(\frac{16 p}{e^{e_{0}}}\right)^{\frac{p}{1-p}}, \quad n_{\mathrm{P}}=\left(\frac{16 p}{e^{e_{0}}}\right)^{\frac{\mathcal{N} p}{1-p}}
$$

Данная неустойчивость связана с тем, что вторая корреляционная длина $\delta_{1}$ в результате роста концентраций как $2 D$-, так и пирловских вихрей сравнивается и становится меньше эффективной длины $\Lambda / \mathcal{N}$. Теперь обе подсистемы вихрей переходят в коллективное состояние, поскольку энергии как $2 D$-, так и пирловских вихрей зависят от их обшей 
концентрации. В этом пределе подсистема $2 D$-вихрей также практически не испытывает влияния пирловских вихрей из-за низкой концентрации последних. Поведение же пирловской подсистемы значительно перестраивается под влиянием $2 D$-вихрей. Температура, при которой появляется неустойчивость пирловской подсистемы относительно диссоциации вихревых диполей, перенормируется за счет взаимодействия с подсистемой $2 D$-вихрей и перестает зависеть от числа слоев $\mathcal{N}$. Без учета влияния подсистемы $2 D$-вихрей эта температура определялась бы из условия $\mathcal{N} p=1$ и имела бы вид

$$
T_{\mathrm{P}}=\frac{\phi_{0}^{2} \mathcal{N}}{16 \pi^{2} \Lambda\left(T_{\mathrm{P}}\right)} .
$$

Она совпадает с температурой появления неустойчивости в однослойной пирловской пленке толшиной $\mathcal{N} d$.

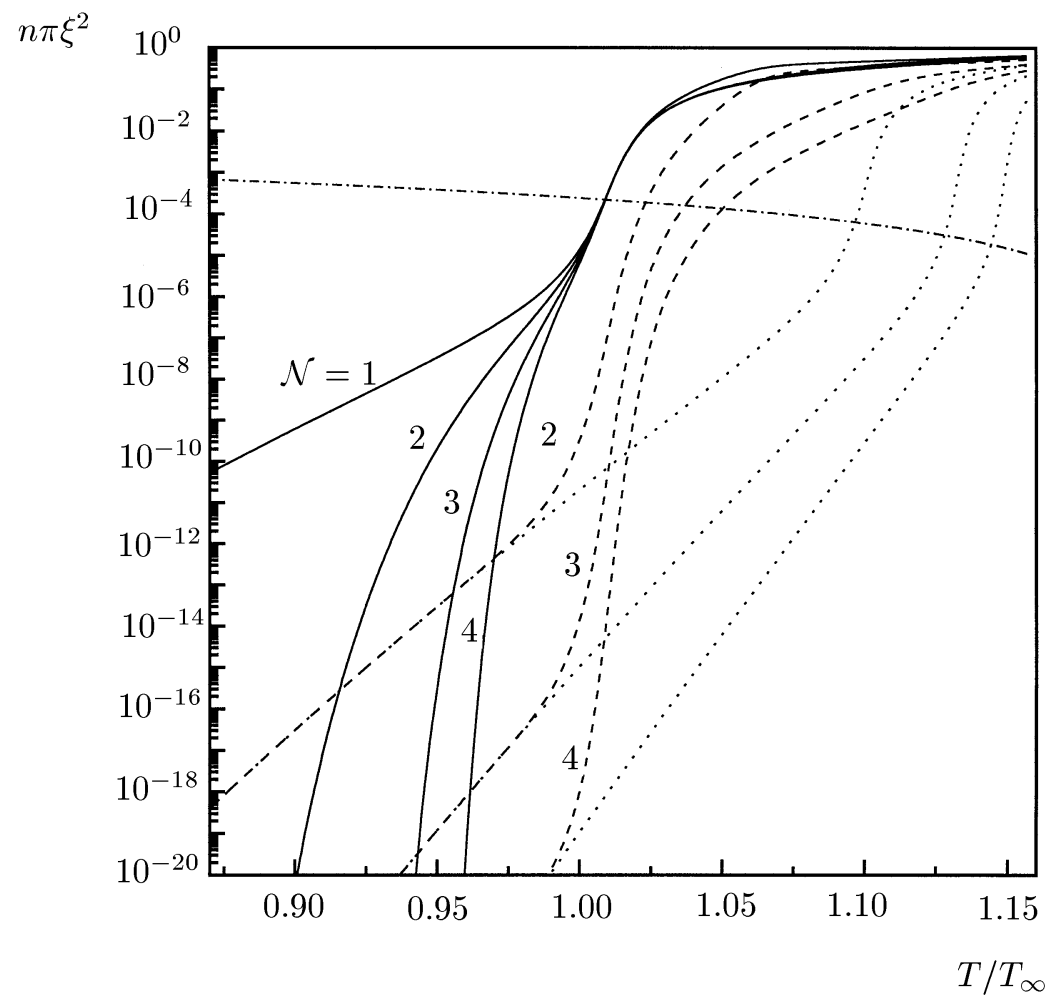

На рисунке показаны найденные численно решения системы уравнений равновесия (26) для сверхпроводников с различным числом слоев $\mathcal{N}$. Сплошными линиями показаны концентрации $2 D$-вихрей, а штриховыми - пирловских. На этих решениях отчетливо видны обе асимптотики. Пунктирной линией показаны температурные зависимости концентрации пирловских вихрей без учета их взаимодействия с $2 D$-вихрями. Штрих-пунктирной линии соответствует концентрация вихрей, при которой $\delta=\Lambda$. Расчеты выполнены для пленок с параметрами $\Lambda / \xi=100, e_{0}=3.3$ и $T_{\mathrm{c}} / T_{\infty}=1.2$.

В случае $\mathcal{N}=1$ наши результаты совпадают с результатами, полученными в работе Рыжова и Тареевой [13] в кольцевом приближении. 


\section{5. ЗАКЛЮЧЕНИЕ}

В данной работе рассмотрены термодинамические свойства системы магнитных вихрей в тонкой слоистой сверхпроводяшей пленке. Слоистая структура этих сверхпроводников определяет такие свойства $2 D$-вихрей, характерные для массивных слоистых сверхпроводников, как логарифмическая расходимость собственной энергии и кулоновский характер взаимодействия. Наличие полей размагничивания вне пленки, связанное с малой ее толщиной, обусловливает нелокальную часть взаимодействия вихрей, характерную для пирловских вихрей.

Такая структура $2 D$-вихрей ведет к более сложным термодинамическим свойствам по сравнению с массивными слоистыми сверхпроводниками. В частности, возникают две температуры, при которых появляются неустойчивости в системе вихрей, характерные для фазового перехода БКТ. Причем одна из них $\left(T_{\mathrm{KT}}\right)$ соответствует реальному переходу в подсистеме $2 D$-вихрей, а вторая $\left(T_{\infty}\right)$ связана со сменой механизма, ответственного за экранирование пирловской части взаимодействия, происходящей без фазового перехода.

В работе имеется также методический аспект. В ней хорошо известная аналогия между статсуммами классического кулоновского газа и скалярной моделью синус-Гордон распространена на целый класс классических частиц с парным взаимодействием, потенциал которого удовлетворяет уравнению вида (14) с дельта-функцией в правой части. Такой подход к статистике классических систем не является совершенно новым (см., например, [14]). Он позволяет для исследования термодинамических свойств классических систем использовать весь арсенал средств, развитых в теории поля.

\section{Список литературы}

[1] J. R. Clem. Phys. Rev. B. 1991. V. 43. P. 7837

[2] S. N. Artemenko, A. N. Kruglov. Phys. Lett. A. 1990. V. 143. P. 485.

[3] В. Л. Березинский. ЖЭТФ. 1971. Т. 61. С. 1144.

[4] J. M. Kosterlitz, D. G. Thouless. J. Phys. C. 1973. V. 6. P. 1181.

[5] V. Cataudella, P. Minnhagen. Physica C. 1990. V. 166. P. 442.

[6] P. Minnhagen. Rev. Mod. Phys. 1987. V. 59. P. 1001.

[7] G. Blatter, M. V. Feigel'man, V.B. Geshkenbein, A.L. Larkin, V. M. Vinokur. Rev. Mod. Phys. 1994. V. 60. P. 1125.

[8] J. Pearl. Appl. Phys. Lett. 1964. V. 5. P. 65.

[9] Р. Балеску. Равновесная и неравновесная статистическая механика. М.: Мир, 1978.

[10] В. С. Капитонов, В. Н. Попов. ТМФ. 1976. Т. 26. С. 246.

[11] В. Н. Попов. Континуальные интегралы в квантовой теории поля и статистической физике. М.: Атомиздат, 1976.

[12] J. Frölich. Quantum sine-Gordon equation and quantum solitons in two space-time dimensions. In: Renormalization Theory: Proceedings of the NATO advanced study Institute held at the international school of mathematical physics. Eds. G. Velo, A.S. Wightman. Dordrecht: Reidel, 1975. P. 371.

[13] V. N. Ryzhov, E. E. Tareyeva. Phys. Rev. B. 1993. V. 49. P. 6162.

[14] A. L. Kholodenko. J. Chem. Phys. 1989. V. 91. P. 4849.

Поступила в редакцию 5.I.2000 г., после доработки 6.VI.2000 г. 